23

\title{
Application of Hilbert Analysis in Orthogonal Fourier Fringe-projection to Improve Object Shape Reconstruction *
}

\author{
(C) Ori Izhak Rosenberg and David Abookasis
}

Department of Electrical and Electronics Engineering, Ariel University, Ariel 407000, Israel

e-mail: davida@ariel.ac.il

Received July 28, 2020

Revised November 11, 2020

Accepted November 26, 2020

Three-dimensional (3D) measurement of an object is widely used in many fields including machine vision, quality control, robotics, medical diagnostics, and others. High-precision 3D surface topography is necessary for describing object shape accurately with high spatial resolution. A combined approach to improve 3D object shape recovery based on Fourier orthogonal fringe projection together with Hilbert transform is proposed and demonstrated. This new idea of combination is highly effective due to the suppressing of background intensity of the deformed fringe pattern while the zero spectrum is extracted precisely and easily. Removing the zero order component leads to increase the visualization and resolution of the measured object. Application of Hilbert processing for object shape recovery in orthogonal Fourier projection domain to improve 3D visualization has not been reported before. The processing framework of this strategy is described in detail. Validation of the proposed method is verified by experiments including visualization of objects with various shapes and sizes. A comparison between profilometry methods is also given which verify better performance in reconstruction of complex objects. 3D reconstruction of flow running at different speeds on a scattering medium with this combined approach is also demonstrated for the first time.

Keywords: 3D shape measurements, orthogonal fringes, Fourier and Hilbert transform, image processing.

* Полный текст статьи опубликован в „Optics and Spectroscopy“ 2021 V. 129. N 5. 